\title{
Study of the hyper-Rayleigh scattering first hyperpolarizability of a chalcone derivative in various solvent media
}

\author{
Adailton N. De Castro, ${ }^{1,2}$ Loyanne C.P. Da Silva, ${ }^{1}$ Francisco A.P. Osório,,${ }^{4,5}$ Basílio Baseia, ${ }^{3}$ Clodoaldo Valverde ${ }^{* 1,2}$ \\ ${ }^{1}$ Laboratório de Modelagem Molecular Aplicada e Simulação (LaMMAS), Campus de Ciências Exatas e \\ Tecnológicas, Universidade Estadual de Goiás, 75001-970, Anápolis, GO, Brazil. \\ ${ }^{2}$ Universidade Paulista, 74845-090, Goiânia, GO, Brazil. \\ ${ }^{3}$ Departamento de Física, Universidade Federal da Paraíba, 58.051-970, João Pessoa, PB, Brazil. \\ ${ }^{4}$ Instituto de Física, Universidade Federal de Goiás, 74.690-900, Goiânia, GO, Brazil. \\ ${ }^{5}$ Escola de Ciências Exatas e da Computação, Pontifícia Universidade Católica de Goiás, 74605-220, Goiânia, GO,
} Brazil.

Received September 04, 2019; accepted December 13, 2019; published December 31, 2019

\begin{abstract}
In this work we investigate the solvent media effects on the nonlinear optical properties of a chalcone derivative, (2E)-3-(3-methylphenyl)-1-(4-nitrophenyl) prop-2-en-1-one (3MPNP), using the polarizable continuum model (PCM) at the DFT/B3LYP/6-311+G(d) level. The behavior of hyper-Rayleigh scattering first hyperpolarizability and average second hyperpolarizability is studied as a function of the dielectric constant of a solvent medium in both dynamic and static cases.
\end{abstract}

The interest in molecules and crystals that exhibit efficient nonlinear optical properties (NLO) has grown substantially in the last two decades [1-9], which is mainly due to their potential applications in optoelectronic and optical devices such as light emitting diodes, photo detectors, solar cells, laser generators, biosensors, optical computing, optical communications and optical storage [1-12]. The development of new materials, whose parameters allow their use in the field of photonics for nonlinear second-order optical applications, requires the knowledge of the nonlinear polarization properties of the constituent molecules [13].

One of the well-established techniques to determine the first hyperpolarizability of organic molecules in a solution is hyper-Rayleigh scattering (HRS). In this technique [14-15] incident light in a fundamental frequency is scattered at the second harmonic frequency that is used to define the symmetry of molecules randomly distributed in a liquid [16]. Hyper-Rayleigh Scattering has also been used to describe nanoparticles [16].

Chalcone derivatives are versatile and interesting substrates for the global chemical enterprise, due to their innumerable applications ranging from biomedical science to materials science. Organic molecules, as chalcone derivatives, are a good alternative to inorganic solids, since they exhibit faster optical responses, lower

*E-mail: valverde@ueg.br preparation costs, and greater flexibility in altering the structure in order to optimize the required properties.

In this work the nonlinear optical properties of the chalcone derivative, (2E)-3-(3-methylphenyl)-1-(4nitrophenyl)prop-2-en-1-one (3MPNP) in various solvents are investigated. The 3MPNP with the molecular formula, $\mathrm{C}_{16} \mathrm{H}_{13} \mathrm{NO}_{3}$ (see Fig. 1) has been synthesized and crystallized by a slow solvent evaporation technique and crystallized in the monoclinic centrosymmetric space group $P 2_{1} / c$ [17].

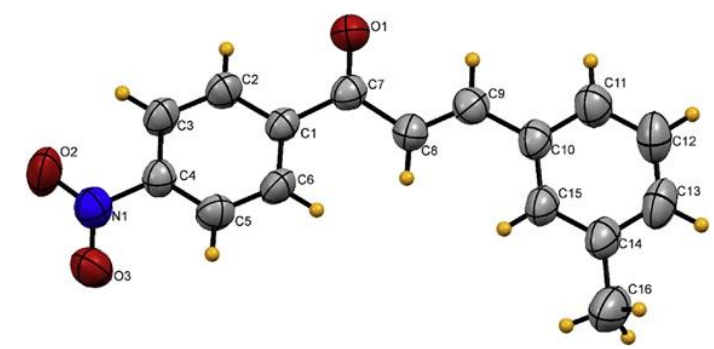

Fig. 1. Molecular structure of 3MPNP showing atom labeling scheme

The geometry optimization of the 3MPNP molecule was performed in gas-phase and in various solvent media using the polarizable continuum model (PCM), where the solvent is modeled as a polarizable and continuum medium, and the cavity of an appropriate shape hosts the solute. The electrostatic interaction solute-solvent is considered through an effective term in the Hamiltonian that is dependent on the macroscopic dielectric constant $(\varepsilon)$. In this work the solvent effects on the dynamic and static electrical parameters of the 3MPNP molecule, as the first and second hyperpolarizability, were considered via the density functional theory (DFT) at PCM/DFT/B3LYP/6-311+G(d) level.

Hyper-Rayleigh scattering (HRS) is the main technique to determine the first hyperpolarizability $\left(\beta_{H R S}\right)$, which is directly related to the second order nonlinear response of an optical material in the molecular level [15]. The HRS 
technique is based on the second harmonic generation (SHG) by molecules dissolved in a solvent medium, with random orientation. The advantage of the HRS technique is that an external electric field is not necessary, making it possible to measure the hyperpolarizabilities of molecules of any structure [18-19].

The HRS first hyperpolarizability $\left(\beta_{H R S}\right)$ of the 3MPNP molecule in a solvent medium can be calculated through the following expression:

$$
\left\langle\beta_{H R S}\right\rangle=\left(\left\langle\beta_{Z Z Z}{ }^{2}\right\rangle+\left\langle\beta_{X Z Z}{ }^{2}\right\rangle\right)^{\frac{1}{2}},
$$

where the propagation direction and polarization of a fundamental light beam is assumed in directions $\mathrm{X}$ and $\mathrm{Z}$ respectively. $\left\langle\beta_{Z Z Z}{ }^{2}\right\rangle$ and $\left\langle\beta_{X Z Z}{ }^{2}\right\rangle$ are the macroscopic means calculated from:

$$
\left\langle\beta_{Z Z Z}^{2}\right\rangle=\sum_{i=1}^{11} C_{i} \delta_{i} \quad \text { and } \quad\left\langle\beta_{X Z Z}^{2}\right\rangle=\sum_{i=1}^{11} D_{i} \delta_{i},
$$

where the coefficients $C_{i}$ and $D_{i}$ are given in Table 1 .

Table 1. Expectation values of macroscopic averages.

\begin{tabular}{cccccccccccc}
\hline & 1 & 2 & 3 & 4 & 5 & 6 & 7 & 8 & 9 & 10 & 11 \\
\hline$C_{i}$ & $\frac{1}{7}$ & $\frac{2}{35}$ & $\frac{2}{35}$ & $\frac{1}{35}$ & $\frac{2}{35}$ & $\frac{1}{35}$ & $\frac{1}{105}$ & $\frac{1}{105}$ & $\frac{2}{105}$ & $\frac{1}{210}$ & $\frac{1}{105}$ \\
$D_{i}$ & $\frac{1}{35}$ & $\frac{4}{105}$ & $-\frac{1}{35}$ & $\frac{3}{35}$ & $-\frac{1}{35}$ & $\frac{2}{105}$ & $\frac{1}{35}$ & $-\frac{1}{210}$ & $-\frac{1}{105}$ & $\frac{1}{70}$ & $-\frac{1}{210}$ \\
\hline
\end{tabular}

The coefficients $\delta_{n}$ are calculated from the components of the first hyperpolarizability $\left(\beta_{i j k}\right)$, see reference [14], and are defined by:

$$
\begin{array}{cc}
\delta_{1}=\sum_{i} \beta_{i i i}^{2}, & \delta_{7}=\sum_{i, j, k} \beta_{i j j} \beta_{i k k}, \\
\delta_{2}=\sum_{i, j} \beta_{i i i} \beta_{i j j}, & \delta_{8}=\sum_{i, j, k}\left(\beta_{j i j}+\beta_{j j i}\right)\left(\beta_{k i k}+\beta_{k k i}\right), \\
\delta_{3}=\sum_{i, j} \beta_{i i i}\left(\beta_{j i j}+\beta_{j j i}\right), & \delta_{9}=\sum_{i, j, k} \beta_{i j j}\left(\beta_{k i k}+\beta_{k k i}\right), \\
\delta_{4}=\sum_{i, j} \beta_{i j j}^{2}, & \delta_{10}=\sum_{i, j, k}\left(\beta_{i j k}+\beta_{i k j}\right)^{2}, \\
\delta_{5}=\sum_{i, j} \beta_{i j j}\left(\beta_{j i j}+\beta_{j j i}\right), & \delta_{11}=\sum_{i, j, k}\left(\beta_{i j k}+\beta_{i k j}\right)\left(\beta_{j j k}+\beta_{j k i}\right) . \\
\delta_{6}=\sum_{i, j}\left(\beta_{j i j}+\beta_{j j i}\right)^{2} &
\end{array}
$$

The average second hyperpolarizability is defined by:

$\langle\gamma\rangle=\frac{1}{15} \sum_{i j=x, y, z}\left(\gamma_{i i j j}+\gamma_{i j j i}+\gamma_{i j i j}\right)$.

Using the Kleinmann symmetry in the static case the equation (2) can simplified to:

$$
\langle\gamma\rangle=\frac{1}{5}\left[\gamma_{x x x x}+\gamma_{y y y y}+\gamma_{z z z z}+2\left(\gamma_{x x y y}+\gamma_{x x z z}+\gamma_{y y z z}\right)\right] .
$$

All computational procedures include the nonlinear response are performed in GAUSSIAN 09 [20].

To increase the knowledge about the 3MPNP compound the effect of a solvent medium on the static and dynamic electric properties of a 3MPNP isolated molecule was considered. It is well known that when immersed in a solvent medium the electrical parameters of organic compounds present important changes, therefore we have considered twelve solvent media and gas-phase, see Table 2 , where $\varepsilon$ is the static dielectric constant of the medium.

In Table 2, the B3LYP/6-311+G(d) static results for the first hyperpolarizability ( $\beta_{H R S}$ in $10^{-30} \mathrm{esu}$ ) and average second hyperpolarizability (in $10^{-36} \mathrm{esu}$ ) for a $3 \mathrm{MPNP}$ isolated molecule in several medium solvents are presented. Also, the results for the gas-phase are shown by comparison.

Table 2. B3LYP/6-311+G(d) results for first static hyperpolarizability (in $10^{-30} \mathrm{esu}$ ) and second static hyperpolarizability (in $10^{-36}$ esu) for 3 MPNP in several solvent media.

\begin{tabular}{lccc}
\hline Medium & $\varepsilon$ & $\left\langle\beta_{H R S}(0 ; 0,0\rangle\right.$ & $\langle\gamma(0 ; 0,0,0)\rangle$ \\
\hline Gas-Phase & 1 & 15.07 & 122.43 \\
Toluene & 2.38 & 26.40 & 232.30 \\
Chloroform & 4.71 & 33.83 & 315.34 \\
Dichloromethane & 8.93 & 38.64 & 372.68 \\
Acetone & 20.9 & 42.24 & 417.65 \\
Ethanol & 24.84 & 42.80 & 424.47 \\
Methanol & 32.61 & 43.40 & 432.31 \\
Acetonitrile & 37.5 & 43.60 & 434.52 \\
DiMethylSulfoxide & 46.7 & 43.99 & 440.16 \\
Formic Acid & 57.9 & 44.11 & 441.69 \\
Water & 78.36 & 44.60 & 447.61 \\
Formamide & 111 & 44.81 & 450.77 \\
n-MethylFormamide- & 182.4 & 45.06 & 454.05 \\
mixture & & & \\
\hline
\end{tabular}

As can be noted from Table 2, the values of all electrical parameters increase with an increase in the static dielectric constant of the solvent medium. From the toluene $(\varepsilon=2.38)$ to n-methylformamide-mixture $(\varepsilon=182.4)$ the values of the electric parameters $\beta_{H R S}(0 ; 0,0)$ and $\langle\gamma(0 ; 0,0,0)\rangle$ show a percentage increasing of $71 \%$ and $95 \%$ respectively. However the increasing of these parameters with the increasing of the dielectric constant value is not linear, as can be seem for $\varepsilon>30$ the effect of the solvent medium exchange cause small changes in the values of the parameters $\beta_{H R S}(0 ; 0,0)$ and $\langle\gamma(0 ; 0,0,0)\rangle$.

In Table 3 we present our results for $\beta_{H R S}(-2 \omega ; \omega, \omega)$, $\gamma(-\omega ; \omega, 0,0)$ and $\gamma(-2 \omega ; \omega, \omega, 0)$ for the electric field frequency, $\omega=0.085$ a.u. $(\lambda=532.3 \mathrm{~nm})$, these electric parameters are related with the second harmonic generation (SHG); dc-Kerr effect and dc-SHG respectively. As can be seen, the variation of the absolute values of the electric parameters does not show a welldefined increase along with an increase in the static dielectric constant of the solvent medium as observed previously in Table 2. Naturally, this fact occurs because the electric dielectric constant is also a function of frequency, $\varepsilon=\varepsilon(\omega)$. 
Figure 2 shows the DFT/B3LYP/6-311+G(d) results for the $\beta_{H R S}$ in both dynamic and static cases as a function of the dielectric constant. As can be seen in the static case, the $\beta_{H R S}$ values increasing monotonically with an increase in the value of the dielectric constant and for $\varepsilon>$ 30 the values saturate. However, the dynamic $\beta_{H R S}$ values present increased oscillations with an increase in $\varepsilon$ values. Also in Fig. 2, the ratio between the macroscopic averages $\left\langle\beta_{X Z Z}{ }^{2}\right\rangle$ and $\left\langle\beta_{Z Z Z}{ }^{2}\right\rangle$ is presented and can be seen - as the static dielectric constant value increases, the ratio $\left\langle\beta_{X Z Z}{ }^{2}\right\rangle /\left\langle\beta_{Z Z Z}{ }^{2}\right\rangle$ decreases from 0.61 to 0.50 and from 0.205 to 0.195 , in the dynamic and static cases respectively.

Table 3. B3LYP $/ 6-311+G(d)$ dynamic results for first hyperpolarizability (in $10^{-30} \mathrm{esu}$ ) and second hyperpolarizability (in $10^{-36} \mathrm{esu}$ ) for $3 \mathrm{MPNP}$ in several medium solvents for $\lambda=$ $532.3 \mathrm{~nm}$.

\begin{tabular}{lccc}
\hline Medium & $\left\langle\beta_{H R S}(-2 \omega ; \omega, \omega)\right.$ & $\gamma(-\omega ; \omega, 0,0)$ & $\gamma(-2 \omega ; \omega, \omega, 0)$ \\
\hline Gas-Phase & 34.88 & 909.30 & -1458.19 \\
Toluene & 59.97 & 2952.19 & -2506.26 \\
Chloroform & 69.99 & 4277.71 & -3345.24 \\
Dichloromethane & 76.82 & 5260.38 & -4539.19 \\
Acetone & 79.57 & 5847.77 & -5984.54 \\
Ethanol & 80.63 & 5983.82 & -6349.36 \\
Methanol & 79.90 & 5988.67 & -6622.24 \\
Acetonitrile & 81.08 & 6097.50 & -6845.75 \\
DMSO & 85.89 & 6529.27 & -7659.98 \\
Formic Acid & 83.61 & 6352.17 & -7494.51 \\
Water & 82.28 & 6290.41 & -7686.64 \\
Formamide & 89.12 & 6858.21 & -8737.03 \\
n-MethylFormamide- & 88.88 & 6858.56 & -8942.83 \\
$\quad$ mixture & & & \\
\hline
\end{tabular}

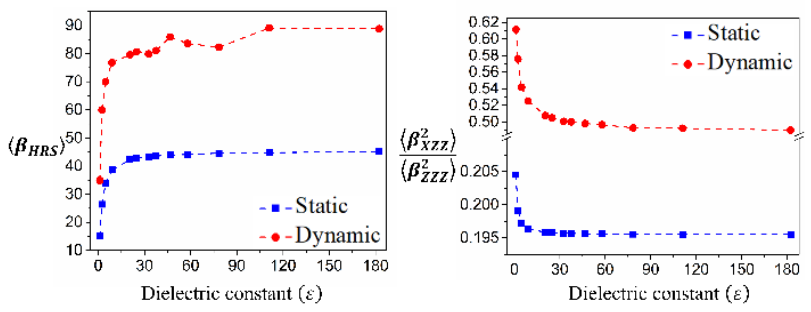

Fig. 2. $B 3 L Y P / 6-311+G(d)$ dynamic results for (a) first hyperpolarizability (in $10^{-30} e s u$ ) (b) $\left\langle\beta_{X Z Z}{ }^{2}\right\rangle /\left\langle\beta_{Z Z Z}{ }^{2}\right\rangle$ for 3MPNP in several medium solvent for $\lambda=532.3 \mathrm{~nm}$.

In conclusion, we have reported a quantum chemical calculation at the PCM/DFT/B3LYP/6-311+G(d) level of the solvent media having an effect on the first and second hyperpolarizabilities of the chalcone derivative (2E)-3-(3methylphenyl)-1-(4-nitrophenyl)prop-2-en-1-one

(3MPNP). The hyper-Rayleigh scattering first hyperpolarizability and the average second hyperpolarizability were calculated in the static and dynamic cases, and their behavior as a function of the value of the dielectric constant for various solvents was studied. We have verified that in static conditions the values of all electrical parameters increase with an increase in the static dielectric constant of the solvent medium. However, for the dynamic case an increase in the electric parameters as a function of the dielectric constant presents oscillations. In DMSO, which is a common solvent medium used in the HRS experiment, the $\beta_{H R S}$ varies from $44 \times 10^{-30} \mathrm{esu}$ (static case) to $86 \times 10^{-30}$ esu (dynamic case); these values are great as compared with the $\beta_{H R S}$ values for other organic compounds [21].

The authors would like to thank the following Brazilian agencies for their financial support: Conselho Nacional de Desenvolvimento Científico e Tecnológico (CNPq), Coordenação de Aperfeiçoamento Pessoal de Nível Superior (CAPES) and Fundação de Apoio à Pesquisa do Estado de Goiás (FAPEG).

\section{References}

[1] L.R. Almeida et al., New J. Chem. 41(4), 1744 (2017).

[2] P.K. Murthy et al., J. Mol. Struct. 1186, 263 (2019).

[3] J. Kongsted, A. Osted, K.V. Mikkelsen, O. Christiansen, J. Chem. Phys. 120(8), 3787 (2004)

[4] T.L. Fonseca, J.R. Sabino, M.A. Castro, H.C. Georg, J. Chem. Phys. 133(14), 1 (2010).

[5] A. Karakaş, Z.E. Koç, M. Fridrichová, P. Němec, J. Kroupa, J. Theor. Comput. Chem. 11(01), 209 (2012).

[6] T. Seidler, K. Stadnicka, B. Champagne, Adv. Opt. Mater. 2(10), 1000 (2014)

[7] L.M.G. Abegão et al., Chem. Phys. Lett. 648, 91 (2016).

[8] W.F. Vaz et al., RSC Adv. 6(82), 79215 (2016).

[9] C. Valverde et al., New J. Chem. 41(19), 11361 (2017).

[10] P.N. Prasad, D.J. Williams et al., Introduction to nonlinear optical effects in molecules and polymers (Wiley, New York 1991).

[11] P. Günter, Nonlinear Optical Effects and Materials (Springer, Berlin Heidelberg 2000)

[12] H. Inan et al., Chem. Soc. Rev. 46(2), 366 (2017).

[13] H.J. Chang et al., J. Korean Phys. Soc. 43(4), 587 (2003).

[14] R. Bersohn, Y. Pao, H.L. Frisch, J. Chem. Phys. 45(9), 3184 (1966).

[15] K. Clays, A. Persoons, Phys. Rev. Lett. 66(23), 2980 (1991).

[16] J. Campo, F. Desmet, W. Wenseleers, E. Goovaerts, Opt. Expr. 17(6), 4587 (2009).

[17] S.R. Prabhu, A. Jayarama, K. Chandrasekharan, V. Upadhyaya, S.W. Ng, J. Mol. Struct. 1136, 244 (2017).

[18] K. Clays, A. Persoons, Rev. Sci. Instrum. 63(6), 3285 (1992).

[19] K. Clays et al., Science 262(5138), 1419 (1993).

[20] M. Frisch et al., Gaussian (Wallingford CT 2009).

[21] J.M.F. Custodio et al., J. Phys. Chem. C 123(10), 5931 (2019). 